\title{
Elpoder explicativo de la ansiedad en los estados de ánimo de deportistas españoles The explanatory power of anxiety in the mood of Spanish athletes
}

*Rafael Peñaloza Gómez, **José Carlos Jaenes Sánchez, *María del Pilar Méndez-Sánchez, ***Patricia Jaenes-Amarillo

*Universidad Nacional Autónoma de México (México), **Universidad Pablo de Olavide(España), ***Universidad de Sevilla (España)

\begin{abstract}
Resumen. La aparición de la ansiedad precompetitiva se ha asociado con múltiples variables tales como la adicción al entrenamiento, las fuentes de motivación extrínsecas, las metas de logro, la edad de los participantes, las habilidades de afrontamiento y la experiencia que se tenga en el deporte. En presente estudio utiliza la perspectiva de la teoría de Rasgo - Estado y la teoría interconductual, para analizar la varianza explicada entre las puntuaciones, del Sport Competition Anxiety Test (SCAT) y el Competitive State Anxiety (CSAI-2), dos instrumentos utilizados en Psicología del Deporte para medir la ansiedad rasgo y estado respectivamente, además se consideró el impacto que tiene la interacción de la Ansiedad Rasgo-Estado en diversos estados de ánimo, medidos mediante el POMS. Se utilizó una muestra de 255 deportistas de diversas disciplinas, de los cuales 168 fueron hombres y 86 mujeres, la media de edad fue 20.46 años ( $\mathrm{DE}=6.08$ años). Los resultados mostraron diferencias significativas en las variables abordadas con respecto al sexo de los participantes y se describen el posible peso de los factores históricos en los situacionales también se reporta el modelo estructural que explica la influencia de la ansiedad rasgo sobre la ansiedad estado y su impacto en los diferentes estados de ánimo de los deportistas.
\end{abstract} Palabras clave: Ansiedad Rasgo, Ansiedad Estado, Deporte, Teoría Interconductual, Estados de Ánimo.

\begin{abstract}
The emergence of pre-competitive anxiety has been associated with multiple variables such as addiction to training, sources of extrinsic motivation, achievement goals, participants' age, coping skills, and experience in sports. In this study we uses the perspective of the Trait - State and inter-behavioral theories to analyze the explained variance between scores of the Sport Competition Anxiety Test (SCAT) and the Competitive State Anxiety (CSAI-2), two instruments used in Sports psychology for measuring trait and state anxiety respectively. In addition, the impact of the interaction of the State-trait anxiety in different moods, measured by POMS, was considered. A sample of 255 athletes (168 men, 86 women; mean age $=20.46$; SD $=6.08$ ) from various disciplines was selected. Results showed significant differences regarding participants' gender. Also, the potential weight of historical and situational factors is described. A structural model explaining the influence of trait anxiety on state anxiety and its impact on different athletes' moods is reported.
\end{abstract}

Keywords: Trait Anxiety, State Anxiety, Sport, Inter-behavioral Theory, Mood States.

\section{Introducción}

El tema de las emociones y de la ansiedad en concreto, ha sido profusamente estudiado en el contexto deportivo desde diversos modelos explicativos(Martens, Vealey, \& Burton, 1990; Casado, 1994; Jaenes, 2000; Ries, Castañeda, Campos, \& Del Castillo, 2012). La ansiedad ha sido definida por Freedson (1991) como un sentimiento subjetivo de aprensión o amenaza percibida, a veces acompañada de un incremento de la actividad fisiológica; ésta suele aparecer en contextos de gran demanda emocional y física; por ello al ser la conducta deportiva una actividad que exige mucho esfuerzo y tenacidad, también es frecuente la presencia de ansiedad, lo que puede llevar al abandono del deporte (Lorenzo, 2001).

Se han identificado múltiples variables que se relacionan con la aparición de la ansiedad precompetitiva, como la adicción al entrenamiento (Arbinaga \& Caracuel, 2005; Zarauz \& Ruiz-Juan, 2013, 2014), las fuentes de motivación extrínsecas (Cecchini, González, \& Contreras, 2004), las metas de logro (Cervelló, Jiménez, Fenoll, Ramos, Del Villar, \& Santos-Rosa, 2002; García-Mas, et al., 2011; Pineda-Espejel, LópezWalle, \& Tomás, 2015; Olmedilla, Andreu, Ortín, \& Blas, 2009; Voigh, Callaghan, \& Ryska, 2000; Ruiz-Juan \& Zarauz, 2012), la edad de los participantes (Hernández, Olmedilla, \& Ortega, 2008), habilidades de afrontamiento (Morillo, Reigal, \& Hernández-Mendo, 2016; Pinto \& Vázquez, 2013), y la experiencia que se tenga en el deporte en el que se compite (Lundqvist, Kenttä, \& Raglin, 2011; Mellalieu, Hanton, \& O'Brien, 2004), estos estudios evidencian principalmente que la percepción de rendimiento, de éxito, además del tipo de deporte que se practique, y las características personales del deportista influyen de una manera u otra en la aparición de la ansiedad (Jaenes, Peñaloza, Navarrete, \& Bohórquez, 2012; Pérez, Hernández, \& García, 2011; Ruiz-Juan, Zarauz, \& Flores-Allende, 2015, 2016).

Igualmente, dentro de los diferentes modelos explicativos, quizás la posible relación rasgo-estado, derivada del modelo teórico de Cattell \& Scheier (1961), sea una de las más abordadas, y que explica como un mismo evento puede provocar diversos niveles de ansiedad en diferen-

Fecha recepción: 05-01-16. Fecha de aceptación: 23-05-16

Rafael Peñaloza Gómez

penaloza.gr@gmail.com tes personas y como, una misma persona, puede experimentar diferentes niveles de ansiedad ante diversos eventos (Ries et al., 2012). La ansiedad estado es un cambio emocional inmediato, modificable por eventos contextuales y el tiempo. Mientras que la ansiedad rasgo se refiere a las diferencias individuales de ansiedad relativamente estables, a tendencias de comportamiento y se puede decir que la ansiedad rasgo no se manifiesta necesariamente en la conducta del individuo, sino más bien se considera que en cuantos y qué eventos, una persona manifiesta síntomas ansiosos (Spielberger, 1972).

De esta forma, la ansiedad precompetitiva es sin duda, uno de los factores más estudiados por su influencia en el rendimiento deportivo, y en muchos casos se ha enfocado en su naturaleza multidimensional (Endler, Kocovsky, \& Macrodimitris, 2001; Cheng, Hardy, \& Markland, 2009, Ries, et al., 2012; Jaenes \& Caracuel, 2005 y 2016), y la forma de medición más frecuente es hacerlo a través de tres dimensiones: ansiedad cognitiva, ansiedad somática y autoconfianza; ésta última, aunque no es parte de la ansiedad, es una variable reflejo, que al estar presente, indica la ausencia de rasgos ansiosos (Andrade, Lois, \& Arce, 2007; Ruiz-Juan, Zarauz, \& Flores-Allende, 2007).

Por ello, se han desarrollado diversos instrumentos para medir las evidencias de la ansiedad; en el caso de la Ansiedad-Estado encontramos al Sport Competition Anxiety Test (SCAT) de Martens (1977), que ha sido adaptado y utilizado en diversos estudios de habla hispana (Guillén \& Álvarez-Malé, 2010; Jaenes, Caracuel y Peréz-Gil, 1999, Olmedilla, et al., 2009). LaAnsiedad-Rasgo ha sido considerablemente investigada mediante el Competitive State Anxiety Inventory-2(CSAI-2)de Martens, Vealey, Burton, Bump, y Smith (1990) adaptado al español por Jaenes, Caracuel, y Pérez-Gil (1999), cuyo uso está ampliamente documentado (Jaenes 2000 y 2002; Janes \& Caracuel; 2016, Jaenes, et al. 2012; Modroño \& Guillén, 2011; Morillo, Reigal, \& Hernández-Mendo, 2016; Olmedilla, et al. 2009; Pineda-Espejel, López-Walle, \& Tomás, 2014 Ramirez, López, Alonso, \& Falcó, 2006).

La postura teórica sobre la ansiedad propone que hay una continuidad entre el rasgo como tendencia y el estado, como respuesta puntual, reactiva a una situación específica; en este caso en la competición deportiva, y como se mencionó anteriormente, diversos estudios se han centrado estudiar en la relación de ansiedad y rendimiento (Cervantes, Rodas, \& Capdevila, 2009; Craft, Magyar, Becker, \& Feltz, 2003; Pinto \& Vázquez, 2013; Scanlan, Babes, \& Scanlan, 2005; Woodman \& Hardy, 2003), encontrando principalmente relaciones negativas entre la 
ansiedad cognitiva y el rendimiento. Sin embargo, el valor explicativo de la unión de los dos constructos no ha sido expuesto claramente desde el punto de vista interconductual.

La teoría interconductual propone que los eventos psicológicos deben investigarse como campos complejos, analizando las diferentes interacciones y los sistemas reactivos de un individuo (Kantor, 1967).

Derivado de lo anterior, el objetivo del presente trabajo es comprender el impacto que tiene la interacción rasgo-estado para el estudio de los estados de ánimo que se presentan en la situación deportiva; además de que es importante saber qué papel juegan los factores de campo situacionales como la experiencia deportiva, la importancia del evento, el nivel competitivo del deportista, etc., y en este trabajo, igualmente hay un intento de entender qué valor explicativo tiene, desde el punto de vista interconductual, la fuerte relación encontrada entre los dos constructos.

\section{Método}

\section{Participantes}

La muestra de este estudio estuvo conformada por 255 deportistas, de los cuales 168 fueron hombres y 86 mujeres, la media de edad de toda la muestra fue 20.46 años con una desviación típica de 6.08 años; la Tabla 1 muestra las diferentes disciplinas que practicaban los participantes.

La media de años de práctica de estos deportistas fue de 7.57 años, con una desviación típica de 4.39 años; también el 29.8\% de ellos mencionaron tener un nivel de competición autonómico, 49\% son competidores de nivel nacional y $18.8 \%$ de nivel internacional (2.4\% no mencionaron su nivel de participación deportiva).

\section{Instrumentos}

Se utilizaron los siguientes instrumentos para recabar los datos que

Tabla 1.

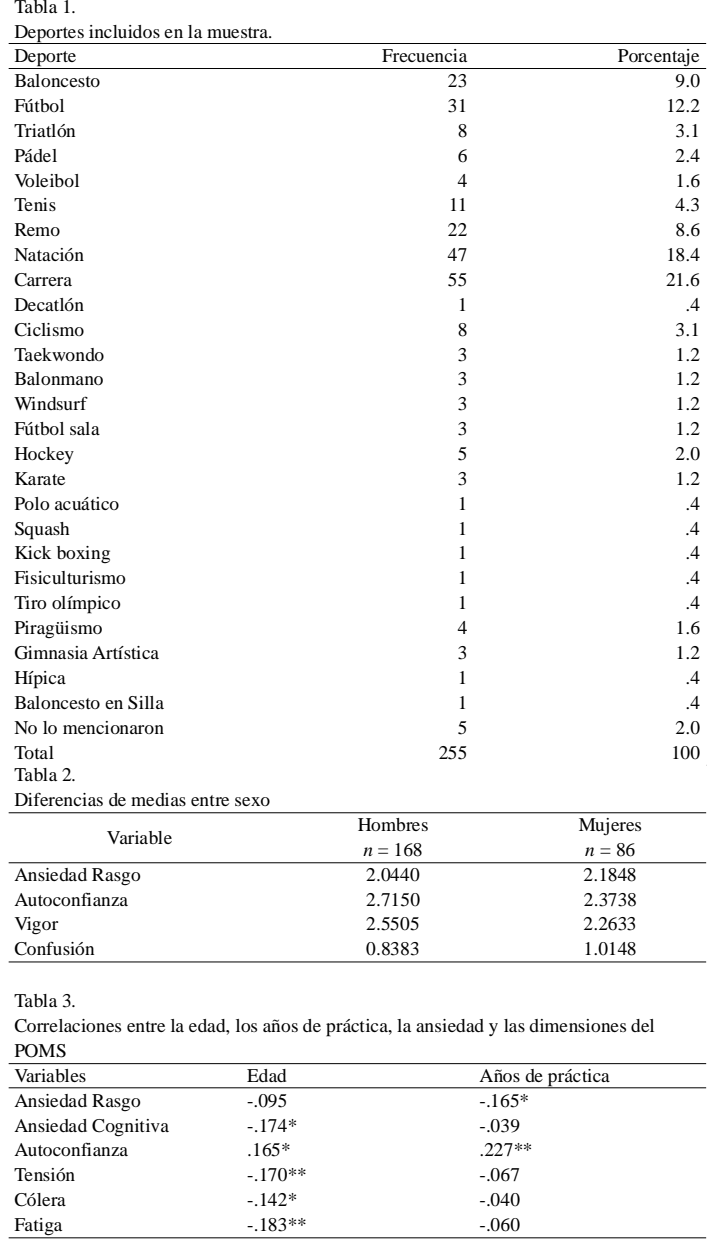

se presentan en el presente estudio:

a) Sport Competition Anxiety Test (SCAT) de Martens (1977) adaptada a población española por Jaenes, Caracuel y Peréz-Gil (1999). Consta de 15 reactivos con tres posibilidades de respuesta y permite evaluar las diferencias individuales en ansiedad rasgo ante la competición. La fiabilidad de esta escala para la presente investigación fue deá $=.784$.

b) Competitive State Anxiety Inventory - 2 (CSAI-2) de Martens, Vealey, Burton, Bump y Smith (1990) en su adaptación española elaborada por Jaenes, Caracuel y Pérez-Gil (1999). Este cuestionario evalúa los componentes cognitivos y somáticos de la ansiedad estado en competición y la autoconfianza en relación a la ejecución deportiva en competición. Consta de 27 reactivos que evalúan tres factores (se muestran los valores de confiabilidad para este estudio): estado de ansiedad cognitivo (á $=.794)$, estado de ansiedad somático (á $=.737)$ y autoconfianza (á $=.813$ ). Se responde sobre cuatro alternativas de respuesta.

c) El cuestionario utilizado en este estudio fue la traducción del POMS (á = .922, para esta investigación) al castellano compuesta por 63 ítems, referidos a los seis estados habituales: Tensión, Depresión, Cólera, Vigor, Fatiga y Confusión (Andrade, 1998).

\section{Procedimiento}

Los deportistas eran usuarios del Centro Andaluz de Medicina del Deporte (CAMD) de Sevilla y acudían a las citas que tenían por diferentes motivos. Se informaba a los mismos del objetivo de la investigación, que era anónima y se les indicaba que su participación era voluntaria y las respuestas serían tratadas de forma confidencial. Los participantes rellenaban los cuestionarios en el mismo Centro, de manera individual y en presencia del psicólogo o del personal de enfermería que había sido previamente adiestrado en la forma de administración.

\section{Resultados}

El análisis de los datos arrojó los siguientes resultados: en primer lugar, se encontraron algunas diferencias significativas por sexo en cuanto a las dimensiones medidas, utilizando una prueba t para muestras independientes y asumiendo varianzas dispares debido a las diferencias de tamaño muestral, los resultados se muestran en la tabla 2.

Además, de acuerdo al nivel de participación (autonómico, nacional e internacional) también se encontraron diferencias significativas en las puntuaciones medias de Ansiedad Rasgo $(F=5.036$, á < .05), entre los deportistas autonómicos (2.1708) y los internacionales (1.9687); Ansiedad Cognitiva $(\mathrm{F}=4.451$, á < .05) entre autonómicos (2.4591) e internacionales (2.2079), y entre estos últimos y los nacionales (2.4435); Ansiedad Somática ( $F=4.354$, á < .05) entre competidores autonómicos (2.2928) e internacionales (2.0018); en Depresión ( $F=3.027$, á < .05) entre deportistas de nivel nacional (.5661) e internacional (.3609); en Vigor ( $F=5$-572, á < .05) entre competidores de nivel autonómico (2.5411)y nacional (2.3328), y entrenacionales einternacionales (2.6339); y finalmente en Confusión $(F=3.699$, á $<.05)$, las diferencias se encontraron entre deportistas de nivel nacional (.9816) e internacional (.7098)

Entre las variables descriptivas del estudio se encontraron también algunas correlaciones entre la edad, los años de práctica y las diferentes dimensiones del SCAT, CSAI-2 y POMS, estas correlaciones se presentan en la tabla 3.

Finalmente se realizó un análisis de ecuaciones estructurales para determinar el tamaño de la influencia que tiene la ansiedad rasgo en la ansiedad estado, y si a su vez, las dimensiones de la ansiedad estado influyen en las dimensiones del POMS. Cabe mencionar que se realizaron pruebas de normalidad siendo Depresión, Cólera y Fatiga las únicas dimensiones que mostraron algunos valores no normales; $\mathrm{y}$ también se comprobó la colinealidad de las variables mediante el índice de DurbinWatson, encontrando un valor que mostró que no había correlaciones seriales entre residuos $(d=1.923)$. El modelo resultante se muestra en la figura 1. 


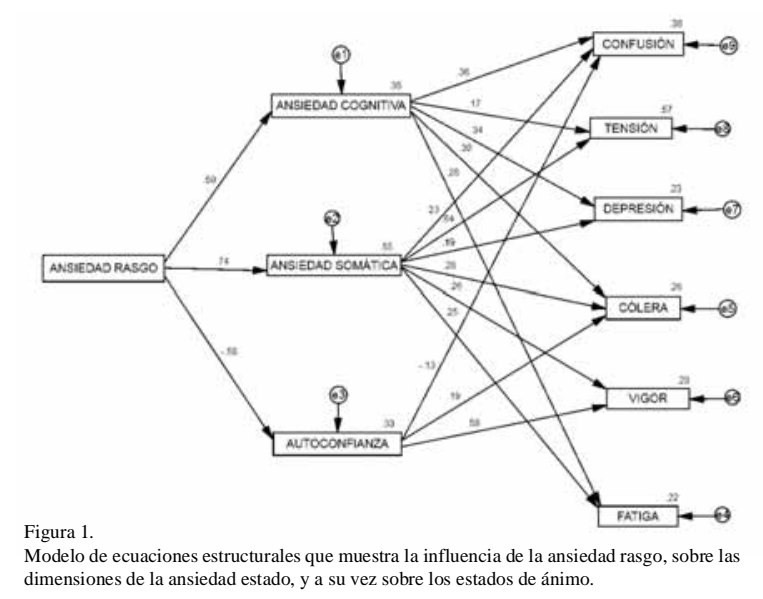

El modelo arrojó índices de ajuste aceptables $(\div 2=19.576$, á > .05; $\mathrm{NFI}=.988, \mathrm{IFI}=.997 ; \mathrm{CFI}=.997 ; \mathrm{RMSEA}=.040, \mathrm{LO} 90: .000, \mathrm{HI}$ 90: .078) y se pueden observar que la ansiedad rasgo explica el $35 \%$ de la varianza de la ansiedad cognitiva, el $55 \%$ de la ansiedad somática y el 33\% de la autoconfianza (en este último caso la influencia es de naturaleza negativa como era de esperar); a su vez las dimensiones del POMS son explicadas por las dimensiones de la ansiedad rasgo, de este modo la Confusión es explicada en un 38\% por la ansiedad cognitiva, ansiedad somática y la autoconfianza, del mismo modo Cólera es influida por las mismas tres variables con una varianza explicada de 26\%. La Depresión (varianza explicada de 23\%), la Tensión (57\%) y la Fatiga (22\%) son influidas por la ansiedad cognitiva y la ansiedad somática; $y$ finalmente el Vigor es ve influido por la ansiedad cognitiva y la autoconfianza, explicado por una varianza del $28 \%$.

\section{Discusión}

Uno de los objetivos de este trabajo fue determinar la influencia de los factores de campo situacionales en la presencia de variaciones emocionales en los deportistas, y aunado a esto, también se encontraron efectos evidentes en cuanto a variables descriptivas de la muestra; esto es diferencias respecto al sexo, donde los hombres puntuaron en promedio más alto que las mujeres en Autoconfianza y Vigor; mientras que las mujeres obtuvieron puntuaciones superiores en Ansiedad Rasgo y Confusión. El que la Ansiedad sea mayor en mujeres está en la línea con lo encontrado por León-Prados, Fuentes y Calvo (2011) y Arbinaga (2013); además, autores como Tabernero y Márquez (1993) ya atribuían a una mayor preocupación de las mujeres por perder reconocimiento debido a una mala actuación.

De la misma forma, en estudios anteriores ya se había señalado una cierta tendencia de las mujeres a puntuar de manera más baja que los hombres en Vigor (Balaguer, Fuentes, Meliá, García-Merita, \& PérezRecio, 1993), pero esta diferencia cambia en función de otras variables como el nivel educativo (Arbinaga, 2013), por lo cual, se puede afirmar que estas diferencias no se deberían de atribuir por si solo al sexo, sin tener en cuenta factores cultural y sociales pertenecientes a la construcción del género.

En cuanto a las relaciones entre la ansiedad rasgo-estado, los niveles de ánimo y los factores de campo situacionales, se encontraron correlaciones negativas entre la edad de los participantes y laAnsiedad Cognitiva, la Tensión, Cólera y Fatiga, y una correlación positiva con la Autoconfianza; esto concuerda con lo encontrado por Hernández, Olmedilla y Ortega (2008), y que indica que los participantes, a mayor edad controlan mejor los efectos emocionales de la situación deportiva.

Además, la relación negativa de la Ansiedad Rasgo con los años de práctica y la relación positiva de esta última con la autoconfianza confirma lo encontrado anteriormente por varios autores (Lundqvist, Kenttä, \& Raglin, 2011; Mellaliu, Hanton, \& O’Brien, 2004; Ruiz-Juan \& Zarauz, 2013) y que pone de manifiesto la importancia de la experiencia en el deporte para la percepción de dominio sobre la disciplina deportiva y el control de los efectos negativos de la ansiedad.
El mismo fenómeno se observa conforme se va a avanzando en el nivel competitivo, y se puede concluir que a medida que el deportista aumenta su nivel de competición, también disminuye su puntuación en variables negativas como la ansiedad somática, la depresión y la confusión; además de que aumenta su vigor.

Con respecto al modelo obtenido es innegable la continuidad entre la ansiedad rasgo y la ansiedad precompetitiva (estado), que se presenta en forma de una influencia directa y significativa del rasgo en el estado y que explica su varianza en gran medida, (tanto la cognitiva como la somática); y que también afecta demanera importante a la autoconfianza del deportista; esta interacción también ha sido comprobada por autores como Ries et al. (2012) y que corrobora la multidimensionalidad de la ansiedad propuesta por Spielberger (1972).

Aunado a esto, tanto la ansiedad cognitiva como la somática, son predictores positivos del facto Cólera, Depresión, Fatiga, Tensión y Confusión; y la ansiedad somática parece predecir de manera positiva el Vigor; y de la misma forma, la Autoconfianza predice positivamente el Vigor y negativamente la Confusión.

Esto, de acuerdo con el modelo interconductual, indica la interacción de la ansiedad rasgo-estado con estados emocionales diversos y que a su vez impactan innegablemente en el rendimiento de los deportistas. A pesar de que estudios como el de García-Mas et al. (2015) y GarcíaMas et al. (2011) indican que la ansiedad somática desaparece antes de la competición y pareciera tener un papel residual, sin embargo, según nuestros hallazgos no debería desecharse su impacto en la salud emocional y afectiva de los deportistas y por ende en la competición.

Finalmente, se recomienda que en futuros estudios se profundizara en las diferencias por sexo respecto a la ansiedad y los estados emocionales, incluyendo variables o factores que pudieran indicar el efecto de los roles de género; de igual forma, se considera sería importante explorar las diferencias por el tipo de deporte en la interacción Ansiedad Rasgo-Estado, algo que no se consideró en el presente estudio debido a las características de la muestra.

\section{Referencias}

Andrade, E. M. F. (1998). Análisis psicométrico del POMS para su aplicación en el ámbito deportivo (Tesis doctoral inédita). Universidad de Santiago de Compostela, España.

Andrade, E. M. F., Lois, G. R., \& Arce, C. F. (2007). Propiedades psicométricas de la versión española del Inventario de Ansiedad Competitiva CSAI-2R en deportistas. Psicothema, 19(1), 150155. Recuperado de http://www.psicothema.com/pdf/3341.pdf

Arbinaga, I. F. (2013). Fisicoculturismo: diferencias de sexo en el estado de ánimo y la ansiedad precompetitiva. Revista de psicología del deporte, 22(2), 353-360. Recuperado de http://ddd.uab.cat/pub/ r e v p side p/revpside p_ a 2013 v 22 n $2 /$ revpsidep_a2013v22n2p353.pdf

Arbinaga, I. F., \& Caracuel, T. J. C. (2005). Precompetición y ansiedad en fisioculturistas. Revista de Psicología del Deporte, 14(2), 195208. Recuperado de http://ddd.uab.cat/record/62955.

Balaguer, I., Fuentes, I., Meliá, J. L., García-Mérita, M. L., \& PérezRecio, G. (1993). El perfil de los estados de ánimo (POMS): Baremo para estudiantes valencianos y su aplicación en el contexto deportivo. Revista de Psicología del Deporte, 4, 39-52. Recuperado de http://ddd.uab.cat/record/63162/

Cattell, R. B., \& Scheier, I. H. (1961). The meaning and measurement of neuroticism and anxiety. New York: Ronald Press.

Casado Morales, M. I. (1994). Ansiedad, stress y trastornos psicofisiológicos (Tesis Doctoral inédita). Universidad Complutense de Madrid, Madrid.

Cecchini, J. A., González, C., \& Contreras, O. (2004). Relaciones entre clima motivacional, la orientación de meta, la motivación intrínseca, la auto-confianza, la ansiedad y el estado de ánimo en jóvenes deportistas. Psicothema, 16(1), 104-109. Recuperado de http:// www.unioviedo.es/reunido/index.php/PST/article/view/8195/8059

Cervantes, J. C., Rodas, G, \& Capdevila, L. (2009). Perfil psicofisiológico de rendimiento en nadadores basado en la variabilidad de la frecuen- 
cia cardíaca y en estados de ansiedad precompetitiva. Revista de psicología del deporte, 18(1). 37-52.

Cervelló, E. M., Jiménez, R., Fenoll, A., Ramos, L., Del Villar, F., \& Santos-Rosa, F. J. (2002). A social-cognitive appproach to the study of coeducation and discipline in Physical Education Classes. SOCIOTAM, Revista Internacional de Ciencias Sociales y Humanidades, 11(2), 43-64.

Cheng, W. N. K., Hardy, L., \& Markland, D. (2009). Toward a threedimensional conceptualization of performance anxiety: Rationale and initial measurement development. Psychology of Sport and Exercise, 10(2), 271-278.

Craft, L. L., Magyar, T. M., Becker, B. J., \& Feltz, D. L. (2003). The relationship between the Competitive State Anxiety Inventory-2 and sport performance: A meta-analysis. Journal of Sport and Exercise Psychology, 25(1), 44-65.

doi:10.1016/ j.psychsport.2008.08.001

Endler, N. S., Kocovsky, N. L., \& Macrodimitris, S. D. (2001). Coping, efficacy and perceived control in acute versus chronic illnesses. Personality and Individual Differences, 30, 617-625. doi:10.1016/ S0191-8869(00)00060-X

Freedson, P. (1991). Dictionary of the sport and exercise sciences. Champaign: Human Kinetics.

García-Mas, A., Fuster-Parra, P., Ponseti, F. J., Palou, P., Olmedilla, A., \& Cruz, J. (2015). Análisis bayesiano de la motivación, el clima motivacional y la ansiedad en jóvenes jugadores de equipo. Anales de psicología, 31(1), 355-366.

García-Mas, A., Leiva, A., Lameiras, J., Jiménez, R., Almeida, P., Smith, R. E., \& Palou Sampol, P. (2011). Ansiedad competitiva y clima motivacional en jóvenes futbolistas de competición, en relación con las habilidades y el rendimiento percibido por sus entrenadores. Revista de psicología del deporte, 20(1), 197-207.

Guillén, F., \& Álvarez-Malé, M. L. (2010). Relación entre los motivos de la práctica deportiva y la ansiedad en jóvenes nadadores de competición. Revista Iberoamericana de Psicología del Ejercicio y el Deporte, 5(2), 233-252.

Hernández, R. G., Olmedilla, A. Z., \& Ortega, E. T. (2008). Ansiedad y autoconfianza de jóvenes judokas en situaciones competitivas de alta presión. Análise Psicológica, 26(4), 689-696.

Jaenes, J. C. (2000). Estado Emocional y Conducta Deportiva: Ansiedad Competitiva en corredores de maratón (Tesis doctoral inédita). Universidad de Sevilla, Sevilla.

Jaenes, J. C. (2002). Entrenamiento psicológico aplicado al remo de competición. En J. Dosil (Ed.) El psicólogo del deporte. Asesoramiento e intervención, Madrid. Síntesis. Pp. 183-205.

Jaenes, J. C., \& Caracuel, J. C. (2005). Maratón: Preparación psicológica para el entrenamiento y la competición. Córdoba: Almuzara.

Jaenes, J. C., \& Caracuel, J. C. (2016). Maratón: Preparación psicológica para el entrenamiento y la competición. Córdoba: Almuzara.

Jaenes, J. C., Caracuel, J. C., \& Pérez Gil, J. A. (Octubre, 1999). Adaptación española del test CSAI-2: un estudio en corredores españoles de maratón. Trabajo presentado en VII Congreso Nacional de la Actividad Física y del Deporte.

Jaenes, J. C., Peñaloza, R. G., Navarrete, K. G. D., \& Bohórquez, M. R. (2012). Ansiedad y autoconfianza precompetitiva en triatletas. Revista Iberoamericana de Psicología del Ejercicio y el Deporte, 7(1), 113-124. Recuperado de https://dialnet.unirioja.es/servlet/ articulo?codigo $=3967899$

Kantor, J. R. (1967). Psicología interconductual. México: Trillas.

León-Prados, J. A., Fuentes, I. G., \& Calvo, A. L. (2011). Ansiedad estado y autoconfianza precompetitiva en gimnastas. RICYDE. Revista Internacional de Ciencias del Deporte, 7(23), 76-91.

Lorenzo, A. (2001). La planificación a largo plazo del deportista dentro del proceso de detección y selección de talentos. Lecturas: Educación Física y Deportes, Revista Digital, 38. Recuperado 22 de Julio, 2009 de http://www.efdeportes.com/efd38/talent.htm.

Lundqvist, C., Kenttä, G., \& Raglin, J. S. (2011). Directional anxiety responses in elite and sub elite young athletes: intensity of anxiety symptoms matters. Scandinavian Journal of Medicine \& Science in Sports, 21(6), 853-862. doi: 10.1111/j.1600-0838.2010.01102.x

Martens, R., Vealey, R. S., \& Burton, D. (1990). Competitive anxiety in sport. Champaign: Human Kinetics.

-210 -
Martens, R., Burton, D., Vealey, R. S., Bump, L. A. y Smith, D. E. (1990). The Competitive State Anxiety Inventory-2 (CSAI-2). En R. Martens, R.S. Vealey y D. Burton (Eds), Competitive Anxiety in Sport. Champaign IL: Human Kinetics.

Mellalieu, S. D., Hanton, S., \& O’Brien, M. (2004). Intensity and direction of competitive anxiety as a function of sport type and experience. Scandinavian Journal of medicine \& science in sports, 14(5), 326-334. doi: 10.1111/j.1600-0838.2004.00389.x

Modroño, C., \& Guillen, F. (2011). Anxiety characteristics of competitive windsurfers: relationships with age, gender, and performance outcomes. Journal of Sport Behavior, 34(3), 281.

Morillo, J. P., Reigal, G. E., \& Hernández-Mendo, A. (2016). Relaciones entre el perfil psicológico deportivo y la ansiedad competitiva en jugadores de balonmano playa. Revista de Psicología del Deporte, 25(1), 121-128.

Olmedilla, A., Andreu, M. D., Ortín, F. J., \& Blas, A. (2009). Ansiedad competitiva y lesiones: factores deportivos y tipos y gravedad de lesión. International Journal of Hispanic Psychology, 2(2).

Pineda-Espejel, H. A., López-Walle, J. M., \& Tomás, I. (2015). Factores situacionales y disposicionales como predictores de la ansiedad y autoconfianza precompetitiva en deportistas universitarios. Cuadernos de Psicología del Deporte, 15(2), 55-70.

Pineda-Espejel, H. A., López Walle, J. M., \& Tomás, I. (2014). Validación de la versión mexicana del CSAI-2R en sus escalas de intensidad y dirección. Revista Mexicana de Psicología, 31(2), 198-212.

Pinto, M. F., \& Vázquez, N. (2013). Ansiedad estado competitiva y estrategias de afrontamiento: su relación con el rendimiento en una muestra argentina de jugadores amateurs de golf. Revista de Psicología del Deporte, 22(1), 47-52.

Ramírez, A., López, M., Alonso, A. I., \& Falcó, F. (2006). Programa de intervención psicológica con árbitros de fútbol. Revista de Psicología del Deporte, 15(2), 311-325.

Ries, F., Castañeda, V. C., Campos, M. D. C., \& Del Castillo, A. O. (2012). Relaciones entre ansiedad-rasgo y ansiedad-estado en competiciones deportivas. Cuadernos de Psicología del Deporte, 12(2), 9-16.

Ruiz-Juan, F., \& Zarauz, A. (2012). Variables que hacen adicto negativamente a correr al maratoniano español. Retos, 21, 38-42. Recuperado de http://retos.org/numero_21/Retos\%2021\%2038-42.pdf

Ruiz-Juan, F., Zarauz, A., \& Flores-Allende, G. (2015). Percepción del éxito en corredores de fondo en ruta en función de variables sociodemográficas. Retos, 27, 136-139. Recuperado de http:// ruizjuan.retos.org/pdf/14_RETOS\%2027\%20136-139.pdf

Ruiz-Juan, F., Zarauz, A., \& Flores-Allende, G. (2016). Percepción del éxito en corredores de fondo en ruta en función de variables de entrenamiento. Retos, 30, 110-113.

Scanlan, T. K., Babkes, M. L., \& Scanlan, L. A. (2005). Participation in sport: A developmental glimpse at emotion. Organized activities as contexts of development: Extracurricular activities, after-school and community programs, 275-309.

Spielberger, C. D. (1972). Conceptual and methodological issues in anxiety research. Anxiety: Current Trends in Theory and Research, 2, 481-493.

Tabernero, B. S., \& Márquez, S. (1993). Diferencias de sexo y edad en los componentes de la ansiedad competitiva. Apuntes: Educación Física y Deportes, (34), 68-73.

Voight, M., Callaghan, J., \& Ryska, T. (2000). Relationship between goal orientation, self-confidence and multidimensional trait anxiety among Mexican-American female youth athletes. Journal of Sport Behavior, 23(3), 271.

Woodman, T., \& Hardy, L. (2003). The relative impact of cognitive anxiety and self-confidence upon sport performance: A metaanalysis. Journal of Sports Sciences, 21(6), 443-457. doi: 10.1080/ 0264041031000101809

Zarauz, A. S., \& Ruiz-Juan, F. (2013). Variables predictoras de la ansiedad en atletas veteranos españoles. Retos, 23, 29-32. Recuperado de https://dialnet.unirioja.es/servlet/articulo?codigo=4135226

Zarauz, A. S., \& Ruiz-Juan, F. (2014). Factores determinantes de la ansiedad en atletas veteranos españoles. Universitas Psychological, 13(3), 1047-1058. Recuperado de http://www.scielo.org.co/pdf/ rups/v13n3/v13n3a20.pdf

Retos, número 30, 2016 ( $2^{\circ}$ semestre) 